- A previous history of alveolar osteitis predisposed the patient to painful post-operative complications following molar or premolar tooth extraction.

- Topical metronidazole gel was ineffective in preventing either alveolar osteitis or the patient returning with pain.

- Complications following tooth extraction are significantly less in those aged 50 years and over compared with those less than 50 years of age.

\title{
The prevention of 'dry socket' with topical metronidazole in general dental practice
}

\author{
D. Reekie, ${ }^{1}$ P. Downes, ${ }^{2}$ C. V. Devlin, ${ }^{3}$ G. M. Nixon ${ }^{4}$ and H. Devlin ${ }^{5}$
}

\begin{abstract}
Objective The purpose of the study was to determine if the intra-alveolar application of topical metronidazole gel could reduce the incidence of alveolar osteitis (dry socket) following routine tooth extraction in molar and premolar extraction sites.

Design This was a multicentre, double blind, randomised, placebocontrolled clinical trial. A total of 302 patients took part, of which 23 returned with alveolar osteitis. Of these, eight had received the metronidazole gel and 15 the placebo.

Setting The study was carried out in three general dental practices by general dental practitioners working in England over the period 20002003.

Main outcome measures Following extraction of either a molar or premolar tooth, either a 25\% metronidazole gel or KY Jelly was syringed gently into the socket. A painful post operative complication was recorded if either a dry socket was present or the patient returned with pain. Results and conclusions The difference in the incidence of alveolar osteitis between the placebo and the active gel groups was not significant and it was concluded that 25\% topical metronidazole gel was not effective in reducing the incidence of alveolar osteitis. It was found that the incidence of alveolar osteitis reduced with increasing age and was more likely to occur in a patient with a previous history of the condition.
\end{abstract}

\section{INTRODUCTION}

Acute alveolar osteitis ('dry socket') is a relatively common complication of the routine extraction of teeth. It is particularly distressing to the patient as it is usually accompanied by severe throbbing pain, with onset 24 to 48 hours after extraction and which can last for up to three weeks. As well as pain this condition is characterised by an empty socket devoid of blood clot with inflamed margins often accompanied by a characteristic odour. Treatment of the condition is by means of irrigation and application of local antiseptic/analgesic dressings such as alvogyl (manufactured by Septodont, Maidstone, UK), or gauze

\footnotetext{
${ }^{1} \mathrm{GDP}, 33$ Herne St., Herne Bay, Herne, Kent, CT6 7HL; ${ }^{2} \mathrm{GDP}, 2$ Nelson Road, Whitstable, Kent, CT5 1DP; 3,4GDP, 41 The Downs, Altrincham, Cheshire, WA14 20G; ${ }^{*}$ Senior Lecturer in Restorative Dentistry, The Dental School, University of Manchester, Higher Cambridge Street, Manchester M156FH

${ }^{*}$ Corresponding Author: Dr Hugh Devlin

Email:Hugh.Devlin@man.ac.uk
}

\section{Refereed paper}

Accepted 28 Feb 2005

doi: $10.1038 /$ sj.bdj.4813253

๑ British Dental Journal 2006; 200: 210-213 soaked in Whitehead's varnish. Even with optimal treatment the pain may take a considerable time to reduce.

Estimates of the incidence of this condition range widely from $0.9 \%$ to $21 \%$ of extractions. ${ }^{1,2}$ The higher figure is reported from studies involving removal of third molars. ${ }^{1}$ Predisposing factors have been stated to be: smoking, the oral contraceptive pill, poor oral hygiene, surgical trauma, gender (females are more commonly affected), a history of previous dry sockets and pre-existing infection. ${ }^{3-5}$

The aetiology of dry socket has been the subject of much debate, with the role of infection under close scrutiny. One study found that patients who developed dry socket had higher preoperative and post-operative ${ }^{6}$ microbial counts than clinically normal patients. Other workers ${ }^{7}$ have implicated particular bacteria such as treponema denticola.

There have been many attempts to find a reliable way to reduce the incidence of dry socket. Anti-fibrinolytic agents have been administered but with little success. ${ }^{8}$ On the whole, antibacterial strategies have shown more promise. Preoperative chlorhexidine mouthwashes have been shown to be moderately effective. ${ }^{1}$ In general, systemic antibiotics have shown mixed results, but metronidazole has shown some success in preventing this condition. ${ }^{9}$ Consistently good results have been obtained with topical antibiotics including clindamycin and tetracycline. ${ }^{10,11}$

The present study was motivated by the desire to prevent the problems caused by dry socket in general practice. Its aim was to develop a simple, easily administered preventive treatment which could be provided safely by general dental practitioners following routine extractions. It was important that it should be quick and simple to administer, did not require extensive pre-operative preparation (eg a week of preoperative mouthwashes) nor the use of systemic antibiotics. Topical antibiotics were thought to offer the greatest potential benefit and metronidazole was selected because of its good safety profile, low risk of allergy and effectiveness against pathogens which cause oral infections. ${ }^{9}$ It has also been show to be effective as a systemic agent.

The study was designed as a double blind, placebo controlled, randomised multi-centre investigation. It was carried out in three general dental practices: two in Kent and one in Greater Manchester. Four general dental practitioners (GDPs) were involved. All practices treated a wide cross section of patients and all provided mixed National Health Service (NHS) and private services. Approval was obtained from the local ethical committees. 


\section{METHODS AND MATERIALS}

In a previous multicentre audit of complications following tooth extraction by the authors, the treatment records of 953 patients were examined. ${ }^{12}$ There was no significant difference between the three dental practices in their dry socket complication rate. From this pilot study, it was estimated that following extraction of non-mobile posterior teeth, $17.5 \%$ of adult patients would be expected to return with pain.

Three hundred and two adult patients were recruited for the study, with informed consent (see sample size calculation). Each patient required one or more routine, non-surgical extraction/s of permanent molar or premolar teeth under local anaesthesia $(2 \%$ lignocaine 1:80,000 adrenaline or 3\% prilocaine with octapressin) Teeth exhibiting a grade 3 or greater mobility were excluded as these were thought to be at very low risk of dry socket because of advanced bone loss. Patients on warfarin, nicoumalone, phenytoin, fluocil, lithium and cimetidine were excluded as there was a potential interaction with metronidazole. Patients were warned to avoid alcohol. Smokers were warned not to smoke during the healing period. Also excluded were pregnant or breast feeding women and people with intellectual impairment. Where more than one extraction was needed only one was randomly chosen to be included in the study. Written, informed consent was obtained from all subjects prior to extraction and application of the gel.

\section{Sample size calculation}

A two-group continuity corrected chi-square test with a 0.050 two-sided significance level will have $90 \%$ power to detect the difference between a test group dry socket proportion of 0.05 and a placebo group proportion of 0.175 (odds ratio of 0.248 ) when the sample size is 148 .

\section{Clinical procedure}

Following the extraction of the tooth, bleeding was controlled and $0.25 \mathrm{ml}$ of $25 \%$ metronidazole gel or placebo gel was gently placed in the socket using a sterile, single use $1 \mathrm{ml}$ syringe with a blunt-ended endodontic irrigation needle. Where the active gel was used, this would introduce $62.5 \mathrm{mg}$ of metronidazole into the socket. The gel displaced the blood clot in the socket. The minimum inhibitory concentration of metronidazole was $<4 \mathrm{mg} / \mathrm{l}$ against a range of gram negative anaerobic bacteria. ${ }^{13}$ We used the minimum concentration of metronidazole in our syringes which would provide a prolonged and effective bacteriocidal dose, despite salivary dilution, and which would also be non-injurious to our patients. The gel was syringed into the base of the extraction socket, displacing the blood clot, and where there was more than one root, equal amounts were introduced into each socket. A sterile gauze swab was placed and the patient instructed to keep it in place for 20 minutes to help retain the dressing. This method of applying the gel was found to be simple and convenient to use by the GDPs taking part in the study.

The patients were provided with some spare swabs and a standardised set of postoperative instructions including advice to phone the surgery if severe pain persisted beyond or developed after 24 hours. Any patient who did call was offered an appointment the same day to see a dentist, the same protocol as used by Rood and Murgatroyd ${ }^{9}$ in their general dental practice-based study. Those taking part in the study were regularly attending patients who were familiar with contacting the dental practice, rather than their medical practitioner. Because not all patients were reviewed, some under-reporting of complications may have occurred.

\section{Randomisation procedure}

The metronidazole and placebo gels were manufactured by Quality Control North West (Stockport, UK). The active gel was made as a suspension of metronidazole in water-soluble KY Jelly and was expected to provide a bacteriocidal release of the antibiotic for several hours after the extraction. The placebo was KY Jelly alone. Metronidazole suspensions have a shelf-life of at least one year, (see http://www.inchem.org), which was much longer than the duration of use of each of our batches of metronidazole. The critical period determining whether a patient develops alveolar osteitis may be within one hour after the extraction; data from Brown et $a l .{ }^{6}$ indicated that patients who developed alveolar osteitis had significantly increased pre-operative and one hour postoperative bacterial numbers. Our gel was present in the sockets and active for this period. Blinding and randomisation was achieved by the manufacturer placing both active and placebo in identical syringes with a label placed to obscure the contents. The syringes were allocated a code number derived from a random number sequence and this number printed on the label. The code was constructed by the manufacturer and was not broken until the end of the study.

The manufacturers carried out bacteriological testing of the syringe contents to ensure sterility, prior to sending them to the GDPs. The syringes were stored in the dental surgeries at $4^{\circ} \mathrm{C}$. Fresh batches of metronidazole and placebo gel were requested every six months and unused gel was discarded.

\section{Main outcome measures}

If the patient displayed the classical signs of a dry socket (blood clot not visible or necrotic, inflamed margin and odour) this was recorded as a dry socket. Some patients returned with pain without a diagnosis of dry socket. Because of the variable clinical presentation of dry socket and its difficulty in diagnosis, a painful post operative complication was recorded if either a dry socket was present or the patient returned with pain. Also recorded was the patient's name, age, tooth notation, gender, and whether there was a history of dry socket, current infection of the tooth, or if the patient was receiving adjunctive antibiotics, arnica, the contraceptive pill or smoked tobacco. The type of local anaesthetic used was also noted.

\section{RESULTS}

One hundred and fifty patients received the placebo and 152 the active metronidazole gel. No serious side effects were reported, but one patient felt nauseous and vomited a few hours after the extraction and two complained of a bitter taste.

The average age of the patient in the study was 49.25 years $(s d=14.763)$, with a range of 18 to 93 years. One hundred and forty males and 162 females took part. Twenty-seven patients (8.9\%) suffered some post-operative pain, necessitating return to the dentists' surgery, and 23 (7.6\%) were diagnosed as 'dry sockets'. The patients were treated in the traditional manner. There was no significant difference between the three practices in the proportion of patients that returned with painful complications $\left(x^{2}=0.827, \mathrm{p}=0.661\right)$ or in the proportion with diagnosed dry socket $\left(x^{2}=0.696, \mathrm{p}=0.706\right)$.

There was no significant difference between the complication rate and whether active or placebo gel was used $\left(x^{2}=1.553, \mathrm{p}=\right.$ 0.213). Of the 23 patients with dry socket, 15 had received the placebo and eight the active gel. There was no statistically significant difference between the proportion of those receiving the active and placebo gels and the occurrence of dry socket $\left(x^{2}=\right.$ $1.781, p=0.182$ )

To examine the effect of the patient's age on the proportion of subjects developing either a painful postoperative complication or a dry socket, the population was divided into young individuals (aged 18-49 years) and older individuals (50-93 years). Twenty subjects aged less than 50 years (13.25\%) developed complications, whereas only seven subjects aged over 50 years $(4.64 \%)$ developed complications $\left.\left(x^{2}=5.857 . \mathrm{p}=0.016\right)\right)$. Similarly, $17 \mathrm{sub}-$ jects aged less than 50 years developed dry socket, and six aged 50 
years and above developed dry socket $\left(x^{2}=4.706, \mathrm{p}=0.03\right)$.

Of those 16 patients with a previous history of dry socket, six $(37.5 \%)$ developed a dry socket in this study. Two hundred and eighty-six patients reported no previous history of dry socket and 17 (5.94\%) of them subsequently suffered dry socket in this study. There was a statistically significant difference in the proportion of each group affected $\left(x^{2}=17.194, \mathrm{p}<0.001\right)$. Therefore, the risk of a patient developing dry socket in our study was not independent of their previous history of dry socket.

Similarly, six of the 27 patients who suffered painful postoperative complications also stated a previous history of dry socket (22\%). Of the 286 patients with no previous history of dry socket, $21(7.34 \%)$ suffered painful complications in our study. There was a significant difference in the proportion of patients with postoperative pain depending on whether they gave a previous history of dry socket or not $\left(x^{2}=13.43, \mathrm{p}<0.001\right)$.

\section{DISCUSSION}

Topical metronidazole gel had no statistically significant effect in reducing the incidence of dry socket in this carefully conducted study, despite many previous investigations showing considerable reductions when other topical antimicrobials were used. ${ }^{10,11,14}$ But there is no expert consensus on its aetiology or the nature of the organisms that are responsible for dry socket. There are several different explanations for the lack of prophylactic activity of metronidazole in our study:

\section{Bacteria play no part in the development of dry socket}

In this hypothesis, tissue damage or impaired wound healing causes dry socket, and bacterial infection is a secondary result. A compromised blood supply, sclerotic bone, or profound vasoconstriction with regional anaesthesia may predispose to dry socket. ${ }^{15,16}$ In our study, a previous history of dry socket was an important factor in determining whether a patient returned with pain. This agrees with work by Berge and Boe, ${ }^{17}$ which indicated that as yet unidentified intrinsic factors influenced post operative healing. The fibrinolytic theory ${ }^{18}$ states that dry socket is the result of loss of the blood clot caused by bone fibrinolysis. Tissue trauma, caused by the extraction, releases tissue activators in the alveolar bone ${ }^{19}$ which diffuse into the blood clot and convert plasminogen to plasmin, a fibrinolytic agent. This dissolves the blood clot releasing kinins which cause pain. Cigarette smoking has been shown to increase the rate of dry socket in some studies, ${ }^{3,20}$ although this was not a significant factor in our study probably because patients were warned not to smoke during the immediate post-operative period. The effect of tobacco smoking may be related to the cytotoxicity of the smoke, the systemic effects of nicotine, or the reduced oxygenation of the healing tissues caused by the carbon monoxide in tobacco smoke. The contamination of the wound site by tobacco smoke may also alter the bacterial population and hence cause a possible increased prevalence of dry socket in smokers.

\section{Metronidazole, active mainly against anaerobes, is ineffective against the organisms which initiate dry socket}

The organisms that are present in normal extraction sockets are S. aureus, S. viridans, C xerosis, Haemophilus parainfluenzae, Fusobacterium species, vibrios and P. melaninogenica. ${ }^{21}$ Except for P melaninogenica and Fusobacterium species, metronidazole has little or no activity against these organisms. Other studies have proposed that reactivated herpes simplex infection may be a cause of dry socket. ${ }^{22}$ Metronidazole would be ineffective against this virus.

Ritzau et $a .^{23}$ in their double-blind, placebo-controlled trial also found that metronidazole was ineffective in preventing alveolar osteitis. They found no preventative effect of a 1,000 mg pre- operative dose of metronidazole given 30 minutes before surgery. Similarly, Barclay ${ }^{24}$ found no significant reduction in the incidence of dry socket when metronidazole was given one hour preoperatively and for three days after the extraction.

\section{Too small a sample size was used in our study}

There is a possibility of publication bias in studies concerning metronidazole, due to editors and reviewers being more willing to accept studies with demonstrated statistical significance. The sample size in our study may have been too small to detect a small beneficial activity of the antibiotic, but other recent double-blind metronidazole studies have used a smaller sample size. ${ }^{25}$ Our study was powered (90\%) to detect a reduction in the proportion of dry sockets in the placebo of 0.175 to 0.05 in the test group, ie that metronidazole has to be very effective otherwise there is little point in recommending its use. With the difference proposed, the number needed to treat for one patient to benefit was eight. However, with the results found (10\% dry socket in the placebo group versus 5\% in the metronidazole group), even if this was significant, the number needed to treat would be 20. This was felt to be too small a benefit, relative to costs, to recommend it for clinical practice.

The additional number of patients required to gain a significant result in our study was calculated using a sample size calculation from the data already collected. A two-group continuity corrected chi-square test with a 0.050 two-sided significance level and 90\% power would require 621 patients in each group to detect the observed difference in the rate of dry socket occurrence in the metronidazole and control groups. The present study took three years to complete and a much larger study involving 1,242 subjects, though desirable, would have been too costly and demanding of practitioners' time.

Of those who returned with pain or where dry socket was diagnosed, the complication rate was higher in the younger age group. Previous studies ${ }^{9,26}$ have also shown that younger people have a higher incidence of dry socket following extraction. Factors such as oral contraceptive use have been shown recently to favour the appearance of dry socket, ${ }^{27}$ and this may be explained by their fibrinolytic activity. However, other studies have found no increased incidence of dry socket with oral contraceptive use. ${ }^{28}$ Our study only included 10 female patients who said they used oral contraceptives and therefore did not include sufficient numbers of female patients taking contraceptives to detect any significant effect of this factor. However, only one patient from this group returned with postoperative pain, but no evidence of a dry socket was present.

\section{CONCLUSION}

Twenty-five per cent metronidazole gel was not effective in reducing the incidence of dry socket. Younger patients and those with a previous history of dry socket were more likely to develop alveolar osteitis.

This study was supported by a grant from the Oral and Dental Research Trust.

1. Larsen P E. The effect of a chlorhexidine rinse on the incidence of alveolar osteitis following the surgical removal of impacted mandibular third molars. J Ora Maxillofac Surg 1991: 49: 932-937.

2. Archer W H. An analysis of 226 cases of alveolalgia. J Dent Res 1939; 18: 256-257.

3. Sweet T B, Butler D P. The relationship of smoking to localized osteitis. J Oral Surg 1979: 37: 732-735.

4. Catellani J E, Harvey S, Erikson S H, Cherkin D. Effect of oral contraceptive cycle on dry socket (localised alveolar osteitis). J Am Dent Assoc 1980; 101: 77-80.

5. Tjernberg A. Influence of oral hygiene measures on the development of alveolar sicca dolorosa after surgical removal of mandibular third molars. Int J Oral Surg 1979; 8: $430-434$

6. Brown LR, Merrill SS, Allen R E. Microbiologic study of introral wounds. J Oral Surg 1970; 28: 89-95.

7. Nitzan D W. On the genesis of dry socket. J Oral Maxillofac Surg 1983; 41: 706-710.

8. Ritzau M. The prophylactic use of tranexamic acid on alveolitis sicca dolorosa. Int J Oral Surg 1973; 2: 196-199. 
9. Rood J P, Murgatroyd J. Metronidazole in the prevention of 'dry socket'. Br J Oral Surg 1979-80; 17: 62-70.

10. Kupfer S R. Prevention of dry socket with clindamycin. N Y State Dent J 1995; 61:30-33.

11. Chapnick $P$, Diamond $L H$. A review of dry socket: A double blind study on the effectiveness of clindamycin in reducing the incidence of dry socket. J Can Dent Assoc 1992; 58: 43-52.

12. Devlin $H$, Reekie $D$, Downes $P$. The aetiology and prevention of dry socket. The GDP 1998; 8-10.

13. Abu-Fanas S H, Drucker D, Hull P S et al. Identification, and susceptibility to seven antimicrobial agents, of $61 \mathrm{Gram}$-negative anaerobic rods from periodontal pockets. J Dent 1991; 19: 46-50

14. Moore J W, Brekke J H. Foreign body giant cell reaction related to placement of tetracycline-treated polylactic acid. J Oral Maxillofac Surg 1990; 48: 808-812.

15. Lehner T. Analysis of one hundred cases of dry socket. Dent Practit Dent Rec 1958 8: $275-279$

16. Meechan J G, Venchard G R, Rogers S N et al. Local anaesthesia and dry socket. A clinical investigation of single extractions in male patients. Int J Oral Maxillofac Surg 1987; 16: 279-284.

17. Berge $T$ । Boe 0 E. Predictor evaluation of post-operative morbidity after surgical removal of mandibular third molar teeth. Acta Odontol Scand 1994; 52: 162-169.

18. Birn H. Aetiology and pathogenesis of fibrinolytic alveolitis (dry socket). Int J Oral Surg 1973; 2: 211-263.
19. Birn H. Bacteria and fibrinolytic activity in 'dry socket'. Acta Odonto/ Scand 1970 28: 773-783.

20. Al-Belasy F. The relationship of 'shisha' (water pipe) smoking to postextraction dry socket. J Oral Maxillofac Surg 2004; 62: 10-14.

21. MacGregor A J, Hart P. Bacteria of the extraction wound. J Oral Surg 1970; 28: 885-887.

22. Hedner $E$, Vahlne $A$, Kahnberg K E, Hirsch J M. Reactivated herpes simplex virus infection as a possible cause of dry socket after tooth extraction. J Oral Maxillofac Surg 1993; 51:370-376.

23. Ritzau M, Hillerup S, Branebjerg P E, Ersbol B K. Does metronidazole prevent alveolitis sicca dolorosa? A double-blind, placebo-controlled clinical study. Int J Oral Maxillofac Surg 1992; 21: 299-302.

24. Barclay J K. Metronidazole and dry socket: prophylactic use in mandibular third molar removal complicated by non-acute pericoronitis. NZ Dent J 1987; 83: 71-75.

25. Bergdahl $M$, Hedstrom L. Metronidazole for the prevention of dry socket after removal of partially impacted mandibular third molar: a randomised controlled trial. Br J Oral Maxillofac Surg 2004; 42: 555-558.

26. MacGregor A J. Aetiology of dry socket. A clinical investigation. Br J Oral Surg 1968; 6: 49-58.

27. Garcia A G, Grana P M, Sampadro F G et al. Does oral contraceptive use affect the incidence of complications after extraction of a mandibular third molar. Br Dent J 2003: 194: 453-455

28. Larsen P E. Alveolar osteitis after surgical removal of impacted mandibular third molar. Identification of the patient at risk. Oral Surg Oral Med Oral Pathol 1992; 73: 393-397. 